\title{
A systematic review of olfactory related questionnaires and scales*
}

\author{
Pengfei Han ${ }^{1,2}$, Tao Su' ${ }^{1}$ Min Qin', Hong Chen ${ }^{1,2}$, Thomas Hummel ${ }^{3}$ \\ 'The Key laboratory of Cognition and Personality, Ministry of Education, Chongqing, China \\ 2 Faculty of Psychology, Southwest University, Chongqing, China \\ ${ }^{3}$ Interdisciplinary Centre Smell and Taste, Department of Otorhinolaryngology, TU Dresden, Dresden, Germany
}

Rhinology 59: 2, 133 - 143, 2021

https://doi.org/10.4193/Rhin20.291

*Received for publication:

June 12,2020

Accepted: August 17, 2020

\begin{abstract}
Purpose: Although neglected by science for a long time, the sense of olfaction has received increasing attention from research areas including psychology, neuroscience, clinical medicine and nutrition. With the rise of psychophysical and neuroimaging research into olfaction, psychometric tools (e.g. questionnaires and scales) are the basis for the quantitative exploration of inter-individual variability regarding olfactory related responses. The current systematic review is to summarize existing olfaction related questionnaires and/or scales.
\end{abstract}

Methods: Peer-reviewed literature on scales and questionnaires related to perception of odors were searched from online databases (PubMed, Web of Science and PsycINFO). Twenty-one articles that meet the following criteria were included in the review: "human species", "no physical odor stimuli" and "describing the original development of the tool", and "with specific focus on olfaction or odor related responses or behaviors". The psychometric properties, advantages and possible disadvantages were discussed.

Results: Existing psychometric measures focus on various aspects of olfactory related responses and behaviors, including affective experiences of odor perception, awareness and attitude towards olfaction, olfactory function and the quality of life change due to olfactory dysfunction, and the ability to create vivid mental odor images. While most of them have been tested to have good reliability and validity, some were relatively time-consuming due to the number of questionnaire items. Besides, although many measures have been used in clinical populations, few have provided information on the predictive validity regarding effectiveness of clinical intervention on changes of certain responses or behaviors.

Summary: The current review provides an overview of olfactory related questionnaires and scales, highlighting the emotional and affective impact of olfaction and the impact on quality of life due to olfactory dysfunction. With growing interest in olfaction as an important sense, the development and use of psychometrically sound measurements in conjunction with objective assessments will advance our understanding of human olfaction and olfactory dysfunction. The review provides a guide for researchers and clinicians alike to select olfactory scales suitable for olfactory research with different experimental purposes and specific samples.

Key words: olfaction, odor, questionnaire, scale, systematic review, psychometrics

\section{Introduction}

Having received relatively little attention over the last 100 years, recent evidence suggests that the human sense of smell is not a negligible entity ${ }^{(1)}$. It is now clear that olfaction has both the hedonic/defense functions and the social functions, influencing behaviors such as food ingestion, harm avoidance, and social communication ${ }^{(2,3)}$. Compared to other senses (e.g. vision or audition), olfaction has a unique intimacy with emotions ${ }^{(4)}$. The olfactory neuroanatomy is intertwined with the brain limbic system which is considered as the primary emotion areas ${ }^{(5)}$. Potent affective responses (e.g. disgust or pleasantness), arise spontaneously in the presence of most olfactory stimuli ${ }^{(6)}$. Recent evidence further promotes the idea that affective valence represents the dominant dimension in olfactory perception, and 
affective experience is the primary behavioral correlate of odor properties ${ }^{(7,8)}$. From a psychological point of view, emotion is a structural element that accompanies and characterizes olfaction and also functionally related to olfaction ${ }^{(9)}$. Emotions can arise from odors and emotions can also influence odor perception (10-12).

In general, olfactory function covers a wide spectrum that includes anosmia (the sense of smell is lost completely or reduced to the extent that the patient has no function that would be useful in daily life), hyposmia (the sense of smell is partially reduced), normosmia (normal olfactory function), and hyperosmia (increased olfactory acuity) ${ }^{(13)}$. Besides, parosmia (distorted odor perceptions in the presence of an odor source) and phantosmia (odor percepts in the absence of an odor) describe qualitative olfactory dysfunction ${ }^{(14)}$. It has been suggested that olfactory dysfunction is a common problem affecting $20 \%$ to $62.5 \%$ of certain populations ${ }^{(15-17)}$. Olfactory dysfunction has a significant impact on quality of life (QoL) ${ }^{(18)}$. Further, it has been argued that both reduced olfactory performance (anosmia/hyposmia) as well as hyperosmia are associated with altered affective or emotional processing. Olfactory dysfunction had been shown to be a biomarker for affective disorders and neurodegenerative diseases, such as depression ${ }^{(19,20)}$, schizophrenia ${ }^{(21)}$, eating disorders ${ }^{(22)}$, or dementia ${ }^{(21,23,24)}$, and also regarded as an early indicator for COVID-19 infection ${ }^{(25)}$.

The last two decades have witnessed an increasing interest in the field of human olfaction. There is high inter-individual variability in olfactory performance ${ }^{(26)}$, affective response to odors ${ }^{(27)}$, odor awareness/reactivity ${ }^{(28)}$, and the importance and application of olfaction. These variabilities could conceivably affect emotions, physiology and behaviors. Assessment of individual differences is critical to provide a better understanding of odor processing and olfactory related behaviors. With the rise of psychophysical and neuroimaging research in olfaction (29), psychometric tools (e.g. questionnaires and scales) appear to survive as a useful, complementary technique, especially for the qualification and interpretation of olfactory related perceptual responses. Along the way, questionnaires or scales that measure different aspects of olfaction have been developed, focusing on the importance of smell, the application of smells in daily life, or the relationship between olfaction and other psychological processes.

The aim of the current review is to provide a synthesis of the existing olfactory scales and questionnaires, to summarize their psychometric properties and correlates, and to evaluate the scales in light of other self-report measures for the assessment of 1) affective or emotional response to odor; 2 ) olfactory awareness and importance; 3) olfactory dysfunction; 4) olfactory imagery. Further, the advantages and disadvantages of these scales are discussed, aiming to provide researchers and clinicians with

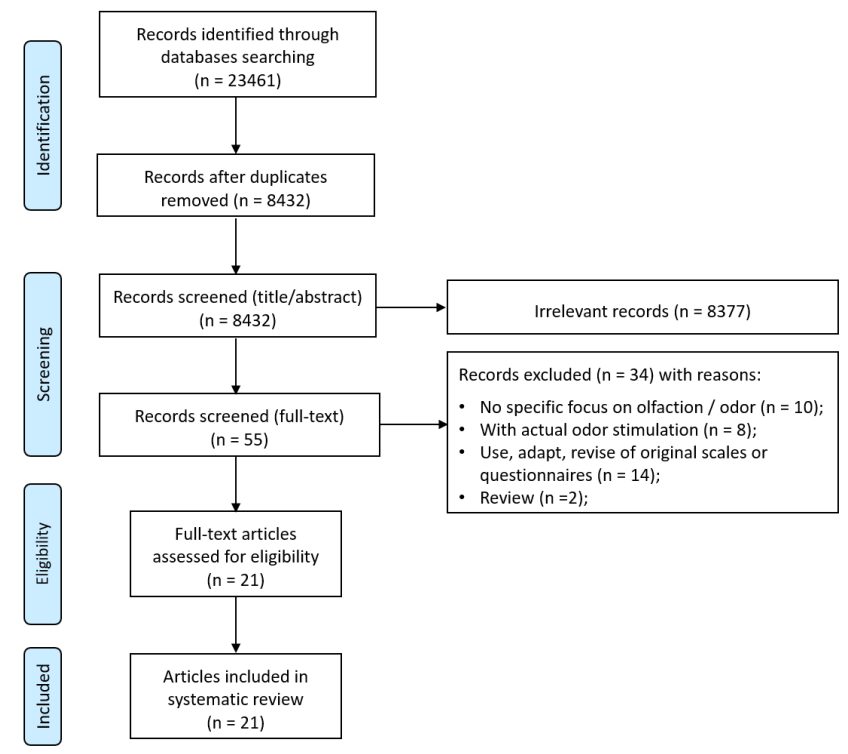

Figure 1. PRISMA flow diagram of the study screening and selection process.

resources regarding self-report measures available of olfaction and to facilitate selection of the optimal measure for a particular clinical or research application.

\section{Materials and methods}

Article search

The initial electronic search was conducted by an author of this article (TS). Three online databases were selected: PubMed, Web of Science and PsycINFO. The following search strategy was used: (olfactory OR olfaction OR smell OR odor OR odour) AND (questionnaire OR scale OR inventory OR test OR score OR measure). Within the database no data filter was applied but the search was limited to human species and English language. A total of 23461 records were generated. Records from different database were exported to EndNoteTM X9. After removal of duplicates, 8423 records were screened based on title, abstract, and key words. 8377 records identified as irrelevant to the review were excluded (e.g. definition of scale was inconsistent with this article, tests with actual odors, etc.). The 55 remaining articles underwent further independent full-text screening by the 2 authors of this review (TS and PH). Articles were considered relevant if both authors rated them accordingly based on the following inclusion criteria: no actual odor stimuli, describing the original development of a measurement tool, with specific focus on olfaction/smell related response or behavior. Finally, twenty-one articles were included for a comprehensive analysis. Figure 1 showed the process of literature search and screening. A summary of the questionnaires included in this review is shown in Table 1. 


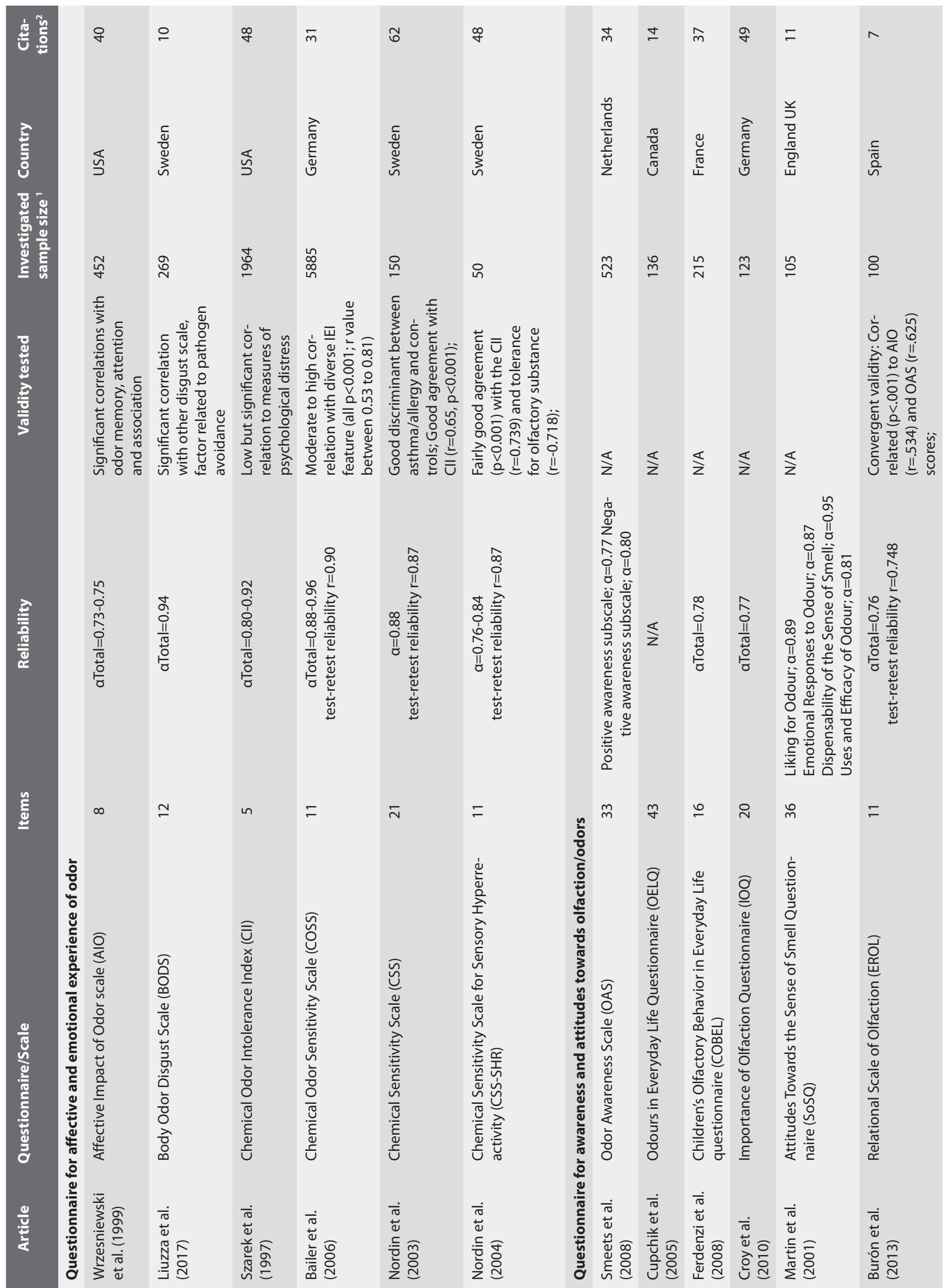




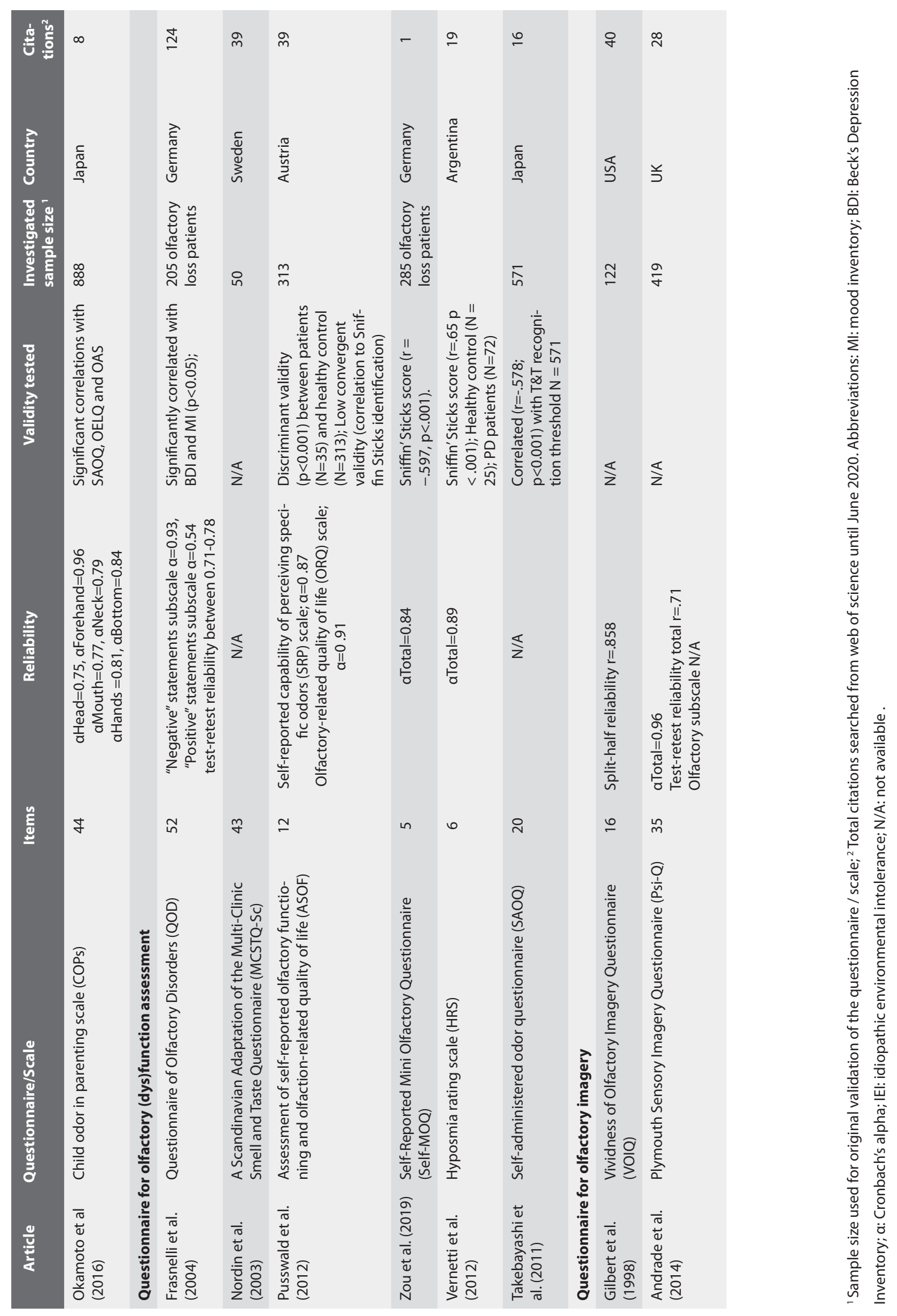




\section{Results}

Affective (valence) and emotional impact of odor One of the most important dimensions for odor perception is the affective response ${ }^{(7,30)}$. Smells perceived as pleasant or unpleasant often serve as potent and direct stimuli to evoke affective states ${ }^{(31,32)}$. The Affective Impact of Odor scale (AIO) was developed to investigate the impact of good or bad smells in determining liking for new food, new places, new cosmetic health products and new persons ${ }^{\left({ }^{33)}\right.}$. The AIO score is calculated by taking the mean of 8 items, with higher scores indicating more impact of odors on liking for the aforementioned topics. The AlO score is correlated with odor-mediated memory, odor attention and odor affect via associations ${ }^{(33)}$, but not related to odor recognition ${ }^{(34)}$. Strengths of the AIO are its brevity and its good reliability among the US and Belgian samples (Cronbach's $a$ of 0.73 and 0.75 , respectively). Another advantage is that the AIO score was shown to be strongly correlated with reactions to odors in an evaluative conditioning experiment, supporting its good convergent validity. Similarly, the Attitudes Towards the Sense of Smell Questionnaire (SoSQ) not only measures the affective impact of odor, but also covers the relative importance and the application of olfaction ${ }^{(35)}$. This measure has the advantage of good reliability for the aggregated items reflecting different aspects attitude towards odor (Cronbach's $a>0.8$ for all). The Odor Awareness Scale (OAS) elaborates the dimensions of awareness, namely the hedonic values toward odors, and evaluates the effects of odors on attention, emotion, memory, product purchase, and the sensitivity and importance of odors (36). The OAS score is positively associated with odor memory (38). Further, the OAS is correlated with actual olfactory function, both in the introductory study and later ones ${ }^{(28,39)}$. OAS accounts for some of the attitudes concerning the value of odors in romantic interest, such as mate selection ${ }^{(40)}$. Current and prior olfactory related behaviors were associated with individual OAS scores ${ }^{(39)}$. Importantly, the OAS score is related to the degree and diversity ${ }^{(41)}$ of odor exposure ${ }^{(42)}$. One advantage of the OAS is the wide coverage of situations with odor, such as eating and drinking, civilization, nature and human. Another advantage is that the OAS has been translated into Italian and Spanish ${ }^{(28,37)}$. One possible disadvantage is the relatively big number of items which could bring burdensome to respondents.

It has been argued that both reduced olfactory performance (anosmia/hyposmia) as well as hyperosmia are associated with altered emotional processing. Self-ratings of odor sensitivity did not correlate with olfactory abilities, but did correlate with "odor annoyance" and the affective importance of odors as measured by the AIO scale ${ }^{(43,44)}$. The chemical odor intolerance index (CII) is a well-validated assessment that requires people to self-report the frequency (almost never, rarely, sometimes, often, almost always) of feeling ill from the odors of five substances (pesticide, paint, perfume, car exhaust and new carpeting), and has pro- mise as a quick and easy screening measure of chemical odor intolerance across a wide range of subjects (younger and older adults, patients with multiple chemical sensitivity condition) ${ }^{(45)}$. The Chemical Odor Sensitivity Scale (COSS) ${ }^{(46)}$ assesses the strong physical responses to odor of common environmental chemicals, providing a useful research tool for assessing chemical sensitivity and promising a brief screening tool for idiopathic environmental intolerance. The Chemical Sensitivity Scale (CSS)

(47) includes questions about the negative affective reactions and behavioral disruptions that individuals experience from environmental exposure to odors or pungent chemical substance. The relatively larger number of items referred to affective reactions (12 out of 22 items) suggests the favor of this facet. In addition, there is a short-version of the CSS, called the Chemical Sensitivity Scale for Sensory Hyper-reactivity (CSS-SHR). This scale has been developed for patients with sensory hyper-reactivity ${ }^{(48)}$. A strength of both the CSS and the COSS is their good testretest reliability ( $r=0.87$ for CSS and $r=0.90$ for COSS). A unique strength of the CSS is that it has been modified and tested in patients with sensory hyper-reactivity, and with normative data available ${ }^{(49)}$. One unique strength of the COSS is that the test is quite stable across time. However, the COSS has limits when attempting to predict olfactory related affective and behavioral consequences for the individuals.

Some body odors are considered to elicit disgust, which is a core emotion that evolved as a defense mechanism, for example to protect the body from potentially harmful substances and diseases ${ }^{(50,51)}$. The Body Odor Disgust Scale (BODS) assesses the trait disgust sensitivity to body odors of sweat, feet, breath, genitals, urine, gas, and feces ${ }^{\left({ }^{52}\right)}$. The scale items were set up as hypothetic scenarios in which participants had to imagine themselves detecting each of these body-generated odors sourced either from themselves or from other people. Participants had to rate to what extent each scenario elicits disgust on a Likert-type scale ranging from 1 ("not disgusting at all") to 5 ("extremely disgusting"). The BODS emphasizes the role of olfaction or body odor in activating behavioral immune system which related to pathogen avoidance ${ }^{(53)}$. One strength of this measure is the good internal consistency (Cronbach's a $>0.90$ ) and its brevity (12 items). Besides, the BODS presented positive correlations with chemical sensitivity scale (CSS) score, olfactory importance score and self-rated olfactory ability ${ }^{(52)}$, supporting its validity. A possible limitation is that the BODS has not been investigated for cross-cultural validity.

\section{Awareness and attitude towards olfaction}

The subjective attitude towards the importance of odors or the olfactory function can be assessed with questionnaires such as the "Odor Awareness Scale" (OAS) ${ }^{(36)}$ or the "Importance of Olfaction Questionnaire" (IOQ) ${ }^{(54)}$. These scales allow to estimate how much people pay attention to chemosensory stimuli in 
their environments. The three subscales of IOQ evaluate the emotions, memories and evaluations associated with odors (Association-scale), the degree of application of smells in daily life (Application-scale), and the importance of smells in decision making (Consequence-scale). The olfactory importance increase with age and was generally higher for females compared to males ${ }^{(55,56)}$. Varied levels of the general attitude as well as the subscale dimensions have been shown in population from different regions ${ }^{(55)}$. However, using selected items from the OAS, there is research demonstrating that the variability in odor awareness is largely due to gender, age and education, but less due to country ${ }^{(57)}$. One strength of the IOQ is the measure of different aspects of attitudes towards odors or olfaction, and inclusion of the aggravation scale to identify the overestimation of olfactory significance among clinical populations (e.g. patients with olfactory loss). For example, the reduced IOQ score among subjects with impaired olfactory function serves as an adaptive coping strategy, while high aggravate symptom among some smelldisordered people may indicate their insufficient coping ${ }^{(58)}$. The Relational Scale of Olfaction (EROL) evaluates the influence of odors on mood, behavior and cognition. With only 11 items, the EROL displayed good psychometric properties by showing an adequate level of test-retest reliability and the acceptable to good convergent validity ${ }^{(59)}$ (Table 1). The Odours in Everyday Life Questionnaire (OELQ) was developed inquiring about the ecological, sexual, social, emotional and memory-related role of odors in everyday life, especially bodily odors ${ }^{(60)}$. However, the OELQ contains several items that are not directly related to odor awareness (e.g., item 22: Do you shave your armpits?). The Children's Olfactory Behavior in Everyday Life questionnaire (COBEL) used food, social, and environmental odors to assess children's attention and use of odors ${ }^{(61)}$. One unique feature of the COBEL is that it is completed in the form of an interview. Besides, it is necessary to control the influence of some factors during the process of interviewing, such as language fluency of the interviewer, and guide the children to answer ${ }^{(41,62)}$. The gender differences revealed by the COBEL had indicated that the stronger odor-orientation of human females is established in childhood ${ }^{(61)}$.

The Child Odor in Parenting scale (COPs) assesses the parents' awareness and experiences of odor from different body parts (head, forehead, hand, mouth, neck and bottom) of their children in daily child care ${ }^{(63)}$. Factor modeling indicated that parental experiences with child odors can be classified into a hygiene care factor (instrumental factor; e.g., perception of the odor evokes the thought of cleaning out the odor source) and an affectionate care factor (affective factor; e.g., perception of the odor evokes sensations of love and reward). Using a group of 888 subjects, the COPs was found to have adequate content validity, concurrent validity, and reliability. Further, the concurrent validity of the COPs was measured by multiple validated olfactory scales (SAOQ, OELQ and OAS). One possible weakness for the COPs is that it penetrates frequent odor experiences, while the unique and infrequent child odor experiences (e.g. odor of sickness) were not included.

The above scales have directly or indirectly measured those aspects (attitude, memory, emotion and applications). Noted that many of the aforementioned scales have been used for clinical populations with psychiatric disorders, such as patients with panic disorder (64), or Autism Spectrum Disorder ${ }^{(65)}$.

\section{Olfactory dysfunction}

The assessment of olfactory performance is a routine test in specialized clinics for olfaction. The most widely used olfactory tests are the "Sniffin' Sticks" battery ${ }^{(66,67)}$ and the University of Pennsylvania Smell Identification test ${ }^{(68)}$. The accuracy of selfreported olfactory performance is limited, but the accuracy is higher among patients with olfactory dysfunction compared to normosmic subjects ${ }^{(69)}$.

The decreased quality of life (QoL) serves as an indicator for olfactory dysfunction ${ }^{(18)}$. A majority of questionnaires ask patients the consequences of olfactory dysfunction and its impact on their daily life, in order to gauge this dimension of olfactory dysfunction. As one of the most widely used questionnaires, the Questionnaire of Olfactory Disorders (QOD) determines the impact of olfactory dysfunction on daily life ${ }^{(70)}$. The QOD consists of 52 statements, which can be divided into 3 domains: 39 negative statements (degree of suffering; NS), 5 positive statements (positive effects and coping strategies) and 8 socially desired statements ("lie scale"). The QOD-NS asks the consequence of olfactory dysfunction on daily life activies, and has been used for olfactory-loss patients due to traumatic brain injury ${ }^{(71)}$ or chronic rhino sinusitis (CRS) ${ }^{(72)}$. Among a group of CRS patients, the worse QOD-NS scores were shown to correlate with lower Sniffin'Sticks TDI score, and also the presence of depression ${ }^{\text {(73). }}$. The minimal clinical important difference (MCID) was developed for the QOD-NS among 128 CRS patients after endoscopic sinus surgery, with an averaged score of 5.2 (with a distribution-based range between 2.6 and 8.6 points) ${ }^{(74)}$. The QOD-NS measures four distinct factors which have differential impact on varying aspects of QoL ${ }^{(75)}$. A short-version using less than half of the questions in the QOD-NS has been validated and proved for the usefulness in clinical settings ${ }^{(76)}$. More recently, the SelfReported Mini Olfactory Questionnaire (Self-MOQ) was developed for quantitative assessment of olfactory dysfunction ${ }^{(77)}$. With only 5 items, this assessment targets mainly the complaints about olfactory problems in daily life. Further, the Self-MOQ is an effective measure with good sensitivity and specificity for discriminating between normosmia and hyposmia (cut-off score 3.5) or anosmia (cut-off score 4.5) (77). A strength of both the OQD and the self-MOQ is that they have good validity had included larger samples for validation. A significant advantage 
of the OQD is that it has been widely referred in other research and has multi-language versions available including English (78) Mandarin ${ }^{(79)}$, Korean ${ }^{(80)}$, and Iranian ${ }^{(81)}$. The strength of the SelfMOQ is that, compared to the OQD, it is much shorter and less burdensome to patients. One possible weakness is the lack of test-retest reliablility information.

The Multi-Clinic Smell and Taste Questionnaire (MCSTQ) was initially developed in the US (also adapted for Scandinavia: MCSTQ-SC) ${ }^{\left({ }^{82}\right)}$. The MCSTQ-Sc explicitly assesses the self-reported odor sensitivity, distorted smell (parosmia) and phantosmia, including the presence, degree and duration of the symptoms. All questions are listed with detailed instruction for responses. Besides, it also contains questions pertaining to the consequences of olfactory dysfunction on daily life and the coping strategies. The MCSTQ had been used for special populations with altered sense of smell, such as pregnant women ${ }^{\left({ }^{83}\right)}$. However, there was no information regarding the validity or reliability for MCSTQ. Similarly, the Cardiff Anomalous Perception Scale (CAPS) ${ }^{(84)}$, contains a few items about the anomalous odor perceptual experience (qualitative and quantitative).

Clinically, subjective testing can be performed using a oneitem question (e.g. do you have a normal olfactory function), single visual analogue scales or Likert questionnaire. However, assessment outcomes using those tools usually showed poor correlation with actual olfactory functions as measured using standard psychophysical tests ${ }^{(85-877}$. Several questionnaires on olfactory dysfunction were developed with detailed quantification of olfactory function, including questions regarding the degree or frequency of odor perception. The Self-Administered Odor Questionnaire (SAOQ), proposed by the Japan Rhinologic Society Committee, contains 20 smell-related questions and asks patients to mark the degree of perception to each of the odor ${ }^{(88,89)}$. Compared to the self-administered olfaction test using a single visual analogue scale (VAS), the SAOQ showed higher sensitivity, specificity and positive and negative predicted values, suggesting its superiority as olfactory test compared to VAS. The five-item self-reported capability of perceiving specific odors scale (one subscale of ASOF) assesses the frequency of odor perception (from very often to never) ${ }^{(90)}$. Similarly, the Hyposmia Rating Scale (HRS), which was initially designed for patients with Parkinson's disease, contains six scenarios in which the patients need to rate the frequency (e.g. always, sometimes, only after being made aware of, and never) of perception ${ }^{(91)}$. In comparison to the single screening question asking whether or not a patient has smell problems, the aforementioned scales are with more clinical relevance. A strength of the aforementioned SAOQ and HRS assessing olfactory functions is that they showed good correlations with T\&T odor threshold and Sniffin' Sticks test scores respectively, which suggested their validity for olfactory function assessments. A weakness for the HRS is the inclusion of a small sample size for its validation, and may not have adequate power for clinically significant. Another weakness is the ceiling effect observed for the HRS which was resulted in decreased specificity. The SAOQ contains odors that are familiar to East Asian people (e.g. soy sauce and steamed rice) which may limit its usefulness among people from other regions. Taken together, in clinical practice, patients can be interviewed or screened for olfactory dysfunction using questionnaires. As a comprehensive olfactory evaluation, it enables systematic evaluation of the patient and yet requires only limited clinical time. The questionnaire can be completed by the patient prior to the clinical visit or during the waiting time between clinical examinations. However, for clinical practices, it is clear that objective olfactory function tests are needed for proper diagnosis. Results from questionnaires are needed to put the clinical results into perspective.

\section{Olfactory imagery}

Olfactory imagery refers to the experience of odor perception without appropriate odor stimulation ${ }^{(92,93)}$. The ability to form olfactory image is mainly reflected by the representational clarity (e.g. vividness of mental images) ${ }^{(94)}$. The Vividness of Olfactory Imagery Questionnaire (VOIQ) measures the olfactory representation ability ${ }^{(96)}$. Screening based on the scale is a simple and effective method to distinguish individuals with high or low olfactory representation abilities. The VOIQ contains 16 scenes with different odors and asks people to evaluate the vividness of the imagined odor using 5-point scale ("1 - perfectly realistic and as vivid as the actual odor" to " 5 - No odor at all, you only know that you are thinking of the odor") (96). Good olfactory imagers (scored higher on the VOIQ) rated pleasant smell as more familiar and had lower anhedonia scores compared to "bad" olfactory imagers, suggesting that individual difference in olfactory imagery vividness is associated with emotions and long-term olfactory memory ${ }^{(97,98)}$. People with good olfactory representations are more likely to recall the pleasure of sensory perception, while those with poor olfactory representations report greater difficulty in recalling pleasant sensory experiences (99). Besides, people with higher olfactory imagery ability show stronger interest in olfaction ${ }^{(100)}$. The ability to create olfactory images is also related to the lexical knowledge of odors, for example, the ability of naming odor. Olfactory experts, who are characterized by a very high level of lexical knowledge of odors, often report good odor imagery ability ${ }^{(14,101)}$. The Plymouth Sensory Imagery Questionnaire (Psi-Q) provides a measure of vividness of imagery across a range of sensory modalities. For olfaction, the Psi-Q asks participants to imagine five different nonfood odors and rate their image on an 11-point scale anchored by 0 (no image at all) and 10 (as vivid as real life) ${ }^{(102)}$. One advantage of the VOIQ and Psi-Q is that they showed good reliability. Besides, the Psi-Q could provide direct comparison of imagery between olfactory and other modalities. Another 
advantage of the Psi-Q is that it has been validated in Spanish ${ }^{(103)}$. One possible disadvantage of the VOIQ is that there is no information reported on the criterion validity.

Olfactory (and visual) imagery are emerged as part of craving processes since substance craving is often accompanied by vivid and frequent olfactory and taste imagery ${ }^{(104,105)}$. Olfactory imagery can influence eating behaviors ${ }^{(106)}$. Patients with peripheral smell loss show a decreased vividness of olfactory representations ${ }^{(108,109)}$. Olfactory mental imagery also interferes with the ability of self-rating one's own olfactory abilities, for example, people with normal (normosmia) or partially reduced (hyposmia) olfactory function maintain that they rely on their ability to imagine odors to evaluate their own olfactory performance ${ }^{(109)}$, although there is conflicting evidence ${ }^{(108)}$.

Recent questionnaires attempt to measure different components of imagery (visual imagery) ability in terms of the underlying sub processes such as image generation, maintenance, inspection, and transformation. However, little progress had been made so far there has been few questionnaires developed on these theory-based sub processes of the olfactory imagery (110). Additionally, a few other questionnaires were developed targeting specific olfactory imagery related behaviors, for example, olfactory dreaming ${ }^{(111)}$. A questionnaire for olfactory dreaming examines the olfactory imagery among people with reported dreaming odors ${ }^{(112)}$.

\section{Discussion}

This review summarized and evaluated the olfactory related psychometric tools (questionnaires and scales). Although most reviewed questionnaires or scales are multifaceted, they were classified into several categories according to the initial purpose of development, namely the affective responses to odors, awareness and attitudes towards olfaction, olfactory function/ dysfunction related quality of life, and olfactory imagery. A close examination of the items/questions and careful consideration of the advantages and disadvantages of the measurement are necessary to determine the best suited measure for the desired purposes. Among the factors relevant to that a decision are the group to be assessed (individuals with normal olfaction or smell dysfunction), or the interest of the study (affective/emotional aspect of olfaction or QoL related issues). In some cases, modifications and validation will be necessary. The validity of reviewed questionnaires/scales is supported by evidences from experimental and longitudinal studies. First, sex difference has been one of the most noteworthy and consistent findings from several psychometric measurements. To illustrate, women compared to men paid more attention to olfactory related cues ${ }^{(61)}$, showed higher interest in the sense of smell and a higher reported importance of olfaction ${ }^{(55,113)}$. This larger interest or importance can be expected to lead to gender differences in olfaction-based decision making and behaviors ${ }^{(114)}$. In the same vein, impairment of olfactory function seems to lead to more complaints in females than in males ${ }^{(70,72)}$. Moreover, females compared to males have significantly higher disgusting sensitivity to body odor ${ }^{(52)}$.

Notwithstanding the intrinsic limitations to many of the psychometric measures, such as sample bias or answers reflecting self-perceived behavior rather than behavior itself, the values of such tools are to provide assessment of the olfactory related behaviors that are difficult to be assessed with other approaches and have had less reflection in the literature. Additionally, those questionnaires/scales can be easily distributed to large population when social distance has to be kept under certain circumstances, the COVID-19 outbreak, for instance. When initially developed, most of the tools were thought to assess individual variations of certain olfactory related behaviors that represent a trait-like individual characteristic that remains stable over time. However, little research had been conducted for their longterm stability. Future work would also benefit from inclusion of more diverse samples (in education, income, race and ethnicity aspects) and multimodal assessments, including a combination of self-report, cognitive processing, behavioral and physiological measurements of olfactory related responses. For example, it is essential to further study the basic neural and cognitive processes underpinning the self-reported scores on psychometric olfactory measurements.

Many of the reviewed questionnaires/scales had been used for clinical populations. For example, in certain people the sense of smell has a high subjective significance when it is impaired (115), while others may not even recognize their olfactory loss (24). Odor awareness was found to be changed in disease such as Autism Spectrum Disorder ${ }^{(65)}$. When repeated measures are conducted, these tools are also useful in characterizing the clinical effect of interventions, including the MCID ${ }^{(74)}$. Given the above issues, these should not be performed in isolation. Rather, when diagnosing olfactory impairment, or assessing the effects of treatment, patient reported outcomes should be used in conjunction with more objective forms of assessment.

\section{Conclusion}

In conclusion, a range of psychometric scales or questionnaires are available tapping on inter-individual differences in olfactory awareness, olfactory affection and emotion, olfactory function/ dysfunctions, and the ability to create olfactory mental images. A review of these psychometric tools could assist psychologists, biologists, clinical scientists, and neuroscientists to select olfactory scales suitable for research with different experimental purposes and specific populations. Especially for clinical practice, questionnaires seem to be a time-efficient and elegant instrument to get an impression of the subjective meaning of the olfactory deficit. Characterization of these features among patients could also help in future interventions, especially to 
improve the olfactory related QoL. As an important complementary approach, such validated assessments could help to achieve a better understanding of the causal path between self-reported olfactory related responses and health effects from odors and their importance in daily life.

\section{Acknowledgements}

None.

\section{Authorship contribution}

$\mathrm{PH}$ : conceptualization and write up; TS: article search and help with write up; MQ: help with write up; HC: edit and review; TH: editing and review.

\section{Conflict of interest}

None.

\section{References}

1. McGann JP. Poor human olfaction is a 19thcentury myth. Science 2017; 356:597.

2. Hummel T, Bensafi M, Nikolaus J, Knecht M, Laing DG, Schaal B. Olfactory function in children assessed with psychophysical and electrophysiological techniques. Behav Brain Res 2007; 180:133-138.

3. Stevenson RJ. An initial evaluation of the functions of human olfaction. Chemical senses 2010; 35:3-20.

4. Calvi E, Quassolo U, Massaia M, Scandurra A, D'Aniello B, D'Amelio P. The scent of emotions: A systematic review of human intra- and interspecific chemical communication of emotions. Brain and behavior 2020:e01585

5. Soudry $Y$, Lemogne C, Malinvaud D, Consoli SM, Bonfils P. Olfactory system and emotion: common substrates. Eur Ann Otorhinolaryngol Head Neck Dis 2011; 128:18-23.

6. Schiffman SS. Physicochemical correlates of olfactory quality. Science 1974; 185:112-117.

7. Khan RM, Luk CH, Flinker A, et al. Predicting odor pleasantness from odorant structure: pleasantness as a reflection of the physical world. J Neurosci 2007; 27:10015-10023.

8. Yeshurun $Y$, Sobel N. An odor is not worth a thousand words: from multidimensional odors to unidimensional odor objects. Ann Rev Psychol 2010; 61:219-241, C211-215.

9. Bochicchio $V$, Winsler A. The psychology of olfaction: A theoretical framework with research and clinical implications. Psychol Rev 2020; 127:442-454.

10. Kadohisa M. Effects of odor on emotion, with implications. Front Syst Neurosci. 2013; 7:66.

11. Kontaris I, East BS, Wilson DA. Behavioral and Neurobiological Convergence of Odor Mood and Emotion: A Review. Front Behav Neurosci 2020; 14:35

12. Flohr EL, Erwin E, Croy I, Hummel T. Sad man's nose: Emotion induction and olfactory perception. Emotion 2017; 17:369-378.

13. Hummel T, Whitcroft KL, Andrews $P$, et al. Position paper on olfactory dysfunction. Rhinology 2016; 56:1-30.

14. Frasnelli J, Landis BN, Heilmann S, et al. Clinical presentation of qualitative olfactory dysfunction. Eur Arch Otorhinolaryngol. 2004; 261:411-415.

15. Murphy C, Schubert CR, Cruickshanks KJ, Klein BE, Klein R, Nondahl DM. Prevalence of olfactory impairment in older adults. JAMA 2002; 288:2307-2312.

16. Bramerson A, Johansson L, Ek L, Nordin S, Bende M. Prevalence of olfactory dysfunction: the skovde population-based study. Laryngoscope 2004; 114:733-737.

17. Schlosser RJ, Desiato VM, Storck KA, et al. A Community-Based Study on the Prevalence of Olfactory Dysfunction. Am J Rhinol Allergy 2020:1945892420922771.

18. Croy I, Nordin S, Hummel T. Olfactory disorders and quality of life--an updated review. Chem Senses 2014; 39:185-194.

19. Taalman H, Wallace C, Milev R. Olfactory Functioning and Depression: A Systematic Review. Front Psychiat 2017: 8:190.

20. Kohli P, Soler ZM, Nguyen SA, Muus JS, Schlosser RJ. The Association Between Olfaction and Depression: A Systematic Review. Chem Senses 2016; 41:479-486.

21. Atanasova B, Graux J, El Hage W, Hommet C, Camus V, Belzung C. Olfaction: a potential cognitive marker of psychiatric disorders. Neurosci Biobehav Rev. 2008: 32:1315-1325.

22. Islam MA, Fagundo AB, Arcelus J, et al. Olfaction in eating disorders and abnormal eating behavior: a systematic review. Front Psychol 2015; 6:1431.

23. Adams DR, Kern DW, Wroblewski KE, McClintock MK, Dale W, Pinto JM. Olfactory Dysfunction Predicts Subsequent Dementia in Older U.S. Adults. J Am Geriatr Soc 2018; 66:140-144

24. Oleszkiewicz A, Kunkel F, Larsson M Hummel T. Consequences of undetected olfactory loss for human chemosensory communication and well-being. Philosophical transactions of the Royal Society of London Series B, Biological sciences 2020; 375:20190265.

25. Lechner M, Chandrasekharan D, Jumani K, et al. Anosmia as a presenting symptom of SARS-CoV-2 infection in healthcare workers - A systematic review of the literature, case series, and recommendations for clinical assessment and management. Rhinology 2020. ;58(4):394-399.

26. Hasin-Brumshtein $Y$, Lancet $D$, Olender $T$. Human olfaction: from genomic variation to phenotypic diversity. Trends Genet. 2009; 25:178-184.

27. Rouby C, Pouliot S, Bensafi M. Odor hedonics and their modulators. Food Quality and Preference 2009; 20:545-549.

28. Demattè ML, Endrizzi I, Biasioli F, Corollaro
ML, Zampini M, Gasperi F. Individual Variability in the Awareness of Odors: Demographic Parameters and Odor Identification Ability. Chemosensory Perception 2011; 4:175-185.

29. Mantel M, Ferdenzi C, Roy JM, Bensafi M. Individual Differences as a Key Factor to Uncover the Neural Underpinnings of Hedonic and Social Functions of Human Olfaction: Current Findings from PET and fMRI Studies and Future Considerations. Brain Topogr. 2019;32(6):977-986.

30. Licon CC, Manesse C, Dantec M, Fournel A, Bensafi M. Pleasantness and trigeminal sensations as salient dimensions in organizing the semantic and physiological spaces of odors. Sci Reports 2018; 8:8444.

31. Dijksterhuis GB, Møller P, Bredie WLP, Rasmussen G, Martens M. Gender and handedness effects on hedonicity of laterally presented odours. Brain Cogn 2002; 50:272-281.

32. Rouby C, Bensafi M. Is there a hedonic dimension to odors? In Olfaction, taste, and cognition. Rouby C, Schaal B, Dubois D, et al. (Eds). New York: NY: Cambridge University Press, 2002.

33. Wrzesniewski A, McCauley C, and Rozin P. Odor and affect: individual differences in the impact of odor on liking for places, things and people. Chem Senses 1999; 24:713-721.

34. Nguyen LA, Ober BA, and Shenaut GK. Odor Recognition Memory: Two Encoding Trials are Better Than One. Chem Senses 2012; 37:745-754.

35. Martin GN, Apena F, Chaudry Z, Mulligan Z, Nixon C. The Development of an Attitudes Towards the Sense of Smell Questionnaire (SoSQ) and a Comparison of Different Professions' Responses. North Am J Psychol 2001; 3:491-502.

36. Smeets MA, Schifferstein HN, Boelema SR, Lensvelt-Mulders G. The Odor Awareness Scale: a new scale for measuring positive and negative odor awareness. Chem Senses 2008; 33:725-734.

37. Burón E, Bulbena A, Pailhez G, Cabré AB. The Spanish version of two olfactory scales: Reliability and validity. Revista de Psiquiatría y Salud Mental (English Edition) 2011; 4:187-194.

38. Arshamian A, Willander J, Larsson M. Olfactory awareness is positively associated to odour memory. J Cognit Psychol 2011; 
23:220-226.

39. Nováková L, Valentova JV, Havlíček J. Engagement in Olfaction-Related Activities is Associated with the Ability of Odor Identification and Odor Awareness. Chemosens Percept 2014; 7:56-67.

40. VanHatten M, Cunningham C, White TL. Olfactory Awareness and the Self-Reported Importance of Olfactory Information in Romantic Interest. Chem. Percept. 2018, 12:40-49.

41. Nováková LM, Fialova J, Havlicek J. Effects of diversity in olfactory environment on children's sense of smell. Sci Reports 2018; 8:2937.

42. Nováková LM, Mrzílková VR. Children's exposure to odors in everyday contexts predicts their odor awareness. Chem. Percept. 2016; 9:56-68.

43. Knaapila A, Tuorila H, Kyvik KO, et al. Selfratings of olfactory function reflect odor annoyance rather than olfactory acuity. Laryngoscope 2008; 118:2212-2217.

44. Knaapila A, Raittola A, Sandell M, Yang B. Self-Ratings of Olfactory Performance and Odor Annoyance Are Associated With the Affective Impact of Odor, but Not With Smell Test Results. Perception 2017; 46:352365.

45. Szarek MJ, Bell IR, Schwartz GE. Validation of a brief screening measure of environmental chemical sensitivity: the chemical door intolerance index. J Environm Psychol 1997; 17:345-351.

46. Bailer J, Witthoft M, Rist F. The Chemical Odor Sensitivity Scale: reliability and validity of a screening instrument for idiopathic environmental intolerance. J Psychosom Res 2006; 61:71-79.

47. 47. Nordin $S$, Millqvist E, Löwhagen $O$ and Bende M. The Chemical Sensitivity Scale: Psychometric properties and comparison with the noise sensitivity scale. Environm Psychol 2003; 23:359-367.

48. Nordin S, Millqvist E, Lowhagen O, Bende M. A short Chemical Sensitivity Scale for assessment of airway sensory hyperreactivity. Int Arch Occup Environ Health 2004; 77:249-254.

49. Nordin S, Bende M, Millqvist E. Normative data for the chemical sensitivity scale. J Environm Psychol 2004; 24:399-403.

50. Rozin P, Fallon AE. A Perspective on Disgust. Psychol Rev 1987: 94:23-41.

51. Davey GC. Disgust: the disease-avoidance emotion and its dysfunctions. Philosophical transactions of the Royal Society of London Series B, Biol Sci 2011; 366:3453-3465.

52. Liuzza MT, Lindholm T, Hawley $C$, et al. The Body Odor Disgust Scale (BODS): Development and Validation of a Novel Olfactory Disgust Assessment. Chem Senses 2017; 42:499-508.

53. Shirasu M, Touhara K. The scent of disease: volatile organic compounds of the human body related to disease and disorder. J Biochem 2011; 150:257-266.

54. Croy I, Buschhüter D, Seo H-S, Negoias S,
Hummel T. Individual significance of olfaction: development of a questionnaire. Eur Arch Otorhinolaryngol. 2010; 267:67.

55. Seo HS, Guarneros M, Hudson R, et al. Attitudes toward Olfaction: A Cross-regional Study. Chem Senses 2011; 36:177-187.

56. Oleszkiewicz A, Walliczek-Dworschak U, Klotze P, Gerber F, Croy I, Hummel T. Developmental Changes in Adolescents' Olfactory Performance and Significance of Olfaction. PLoS One 2016; 11:e0157560.

57. Sorokowska A, Groyecka A, Karwowski M, et al. Global Study of Social Odor Awareness. Chem Senses 2018; 43:503-513.

58. Murr J, Hummel T, Ritschel G, Croy I. Individual Significance of Olfaction: A Comparison Between Normosmic and Dysosmic People. Psychosomatics 2018; 59:283-292.

59. Buron E, Bulbena A, Barrada JR, Pailhez G EROL scale: a new behavioural olfactory measure and its relationship with anxiety and depression symptoms. Actas Esp Psiquiatr 2013; 41:2-9.

60. Cupchik GC, Phillips K, Truong H. Sensitivity to the cognitive and affective qualities of odours. Cognition Emotion 2005; 19:121131.

61. Ferdenzi C, Coureaud G, Camos V, Schaal B. Human awareness and uses of odor cues in everyday life: Results from a questionnaire study in children. Int J Behav Develop 2008; 32:422-431

62. Nováková LM, Havlicek J. Development of odour awareness in pre-schoolers: A longitudinal study. Physiol Behav 2019; 204:224233.

63. Okamoto M, Shirasu M, Fujita R, Hirasawa Y, Touhara K. Child Odors and Parenting: A Survey Examination of the Role of Odor in Child-Rearing. PLoS One 2016; 11:e0154392.

64. Buron E, Bulbena A, Bulbena-Cabre A, Rosado S, Pailhez G. Both anxiety and joint laxity determine the olfactory features in panic disorder. Psych Res 2018; 262:420-426.

65. Kumazaki H, Okamoto M, Yoshimura Y, et al. Brief Report: Odour Awareness in Young Children with Autism Spectrum Disorders. J Autism Dev Disord 2018.

66. Kobal G, Hummel T, Sekinger B, Barz S, Roscher S, Wolf S. " Sniffin'sticks": screening of olfactory performance. Rhinology 1996; 34:222-226.

67. Hummel $T$, Sekinger $B$, Wolf SR, Pauli E, Kobal G. 'Sniffin' Sticks': Olfactory performance assessed by the combined testing of odor identification, odor discrimination and olfactory threshold. Chem Senses 1997; 22:39-52.

68. Doty RL, Shaman P, Dann M. Development of the University of Pennsylvania Smell Identification Test: a standardized microencapsulated test of olfactory function. Physiol Behav 1984; 32:489-502.

69. Lotsch J, Hummel T. Clinical Usefulness of Self-Rated Olfactory Performance-A Data Science-Based Assessment of 6000 Patients. Chemical senses 2019; 44:357-364.
70. Frasnelli J, Hummel T. Olfactory dysfunction and daily life. Eur Arch Otorhinolaryngol. 2005; 262:231-235.

71. Ahmedy F, Mazlan M, Danaee M, Abu Bakar MZ. Post-traumatic brain injury olfactory dysfunction: factors influencing quality of life. Eur Arch Otorhinolaryngol 2020.

72. Simopoulos $E$, Katotomichelakis $M$, Gouveris H, Tripsianis G, Livaditis M, Danielides V. Olfaction-associated quality of life in chronic rhinosinusitis: adaptation and validation of an olfaction-specific questionnaire. Laryngoscope 2012; 122:1450-1454.

73. Mattos JL, Schlosser RJ, Storck KA, Soler ZM. Understanding the relationship between olfactory-specific quality of life, objective olfactory loss, and patient factors in chronic rhinosinusitis. Int Forum Allergy Rhinol 2017; 7:734-740

74. Mattos JL, Schlosser RJ, Mace JC, Smith TL, Soler ZM. Establishing the minimal clinically important difference for the Questionnaire of Olfactory Disorders. Int Forum Allergy Rhinol 2018; 8:1041-1046.

75. Mattos JL, Schlosser RJ, DeConde AS, et al. Factor analysis of the questionnaire of olfactory disorders in patients with chronic rhinosinusitis. Int Forum Allergy Rhinol 2018; 8:777-782.

76. Mattos JL, Edwards C, Schlosser RJ, et al. A brief version of the questionnaire of olfactory disorders in patients with chronic rhinosinusitis. Int Forum Allergy Rhinol 2019. 9:1144-1150.

77. Zou LQ, Linden L, Cuevas M, et al. Selfreported mini olfactory questionnaire (SelfMOQ): A simple and useful measurement for the screening of olfactory dysfunction. Laryngoscope 2019.

78. 78. Langstaff L, Pradhan N, Clark A, et al. Validation of the olfactory disorders questionnaire for English-speaking patients with olfactory disorders. Clin Otolaryngol 2019; 44:715-728.

79. Yang D, Wang J, Ni D, Liu J, Wang $X$. Reliability and validity of the Chinese version of the questionnaire of olfactory disorders (QOD) when used with patients having olfactory dysfunction. Eur Arch Otorhinolaryngol. 2016; 273:3255-3261.

80. Choi WR, Jeong HY, Kim JH. Reliability and validity of the Korean version of the Questionnaire of Olfactory Disorders. Int Forum Allergy Rhinol 2018; 8:1481-1485.

81. Jalessi M, Kamrava SK, Amini E, et al. Is the Persian Version of the "Olfactory Disorder Questionnaire" Reliable and Valid? Iran J Otorhinolaryngol 2017; 29:209-213.

82. Nordin S, Bramerson A, Murphy C, Bende M. A Scandinavian adaptation of the MultiClinic Smell and Taste Questionnaire: evaluation of questions about olfaction. Acta Otolaryngol 2003; 123:536-542.

83. Nordin S, Broman DA, Olofsson JK, Wulff M. A longitudinal descriptive study of selfreported abnormal smell and taste perception in pregnant women. Chem Senses 2004; 29:391-402. 
84. Bell V, Halligan PW, Ellis HD. The Cardiff Anomalous Perceptions Scale (CAPS): a new validated measure of anomalous perceptua experience. Schizophrenia bulletin 2006: 32:366-377.

85. Philpott CM, Wolstenholme CR, Goodenough PC, Clark A, Murty GE. Comparison of subjective perception with objective measurement of olfaction. Otolaryngol Head Neck Surg 2006; 134:488490.

86. Landis BN, Hummel T, Hugentobler M, Giger $R$, and Lacroix JS. Ratings of overall olfactory function. Chem Senses 2003; 28:691-694.

87. Doty RL, McKeown DA, Lee WW, Shaman P. A study of the test-retest reliability of ten olfactory tests. Chem Senses 1995; 20:645656.

88. Tsuzuki K, Fukazawa K, Takebayashi H, et al. Olfactory evaluation using a self-administered odor questionnaire. Nihon Bika Gakkai Kaishi (Jap J Rhinol) 2009; 48:1-7.

89. Takebayashi H, Tsuzuki K, Oka H, Fukazawa K, Daimon T, Sakagami M. Clinical availability of a self-administered odor questionnaire for patients with olfactory disorders. Auris Nasus Larynx 2011; 38:65-72.

90. Pusswald G, Auff E, Lehrner J. Development of a Brief Self-Report Inventory to Measure Olfactory Dysfunction and Quality of Life in Patients with Problems with the Sense of Smell. Chem. Percept. 2012; 5:292-299.

91. Millar Vernetti P, Perez Lloret S, Rossi M Cerquetti D, Merello M. Validation of a new scale to assess olfactory dysfunction in patients with Parkinson's disease. Parkinsonism Relat Disord 2012; 18:358-361.

92. Nanay B. Multimodal mental imagery. Cortex 2018; 105:125-134.

93. Stevenson RJ, Case TI. Olfactory imagery: a review. Psychon Bull Rev. 2005; 12:244-264.

94. Palmiero M, Piccardi L, Giancola M, Nori R, D'Amico S, Olivetti Belardinelli M. The format of mental imagery: from a critical review to an integrated embodied representation approach. Cogn Process. 2019; 20:277-289.

95. Arshamian A, Larsson M. Same same but different: the case of olfactory imagery. Front Psychol 2014; 5:34.

96. Gilbert AN, Crouch M, Kemp SE. Olfactory and visual mental imagery. J Ment Imag. 1998; 22:137-146.

97. Bensafi M, Rouby C. Individual differences in odor imaging ability reflect differences in olfactory and emotional perception. Chem Senses 2007; 32:237-244.

98. Rouby C, Bourgeat F, Rinck F, Poncelet J, Bensafi M. Perceptual and sensorimotor differences between "good" and "poor" olfactory mental imagers. Ann New York Acad Sci. 2009; 1170:333-337.

99. Bensafi M, Sobel N, Khan RM. Hedonicspecific activity in piriform cortex during odor imagery mimics that during odor perception. J Neurophysiol 2007; 98:3254-3262.

100. Arshamian A, Olofsson JK, Jönsson FU, Larsson M. Sniff Your Way to Clarity: The Case of Olfactory Imagery. Chem Percept. 2008; 1:242-246.

101.Plailly J, Delon-Martin C, Royet JP. Experience induces functional reorganization in brain regions involved in odor imagery in perfumers. Hum Brain Mapp 2012; 33:224-234.

102. Andrade J, May J, Deeprose C, Baugh SJ, Ganis G. Assessing vividness of mental imagery: The Plymouth Sensory Imagery Questionnaire. Brit J Psychol 2014; 105:547563

103. Pérez-Fabello MJ, Campos A. Spanish Version of the Plymouth Sensory Imagery Questionnaire. Front Psychol 2020; 11.

104. Tiggemann M, Kemps E. The phenomenology of food cravings: the role of mental imagery. Appetite 2005; 45:305-313.

105. Harvey K, Kemps E, Tiggemann M. The nature of imagery processes underlying food cravings. Br J Health Psychol 2005; 10:49-56.

106. Krishna A, Morrin M, Sayin E. Smellizing Cookies and Salivating: A Focus on Olfactory Imagery. J Consum Res 2014 . 41:18-34.

107. Patel BP, Aschenbrenner K, Shamah D, Small DM. Greater perceived ability to form vivid mental images in individuals with high compared to low BMI. Appetite 2015; 91:185-189.

108. Flohr EL, Arshamian A, Wieser MJ, et al. The fate of the inner nose: odor imagery in patients with olfactory loss. Neurosci 2014;
268:118-127.

109. Kollndorfer K, Kowalczyk K, Nell S, Krajnik J, Mueller CA, Schopf V. The inability to self-evaluate smell performance. How the vividness of mental images outweighs awareness of olfactory performance. Front Psychol 2015; 6:627.

110. Lacey S, Lawson R. Imagery Questionnaires: Vividness and Beyond. Imagery questionnaires: Vividness and beyond. In S. Lacey \& R. Lawson (Eds.), Multisensory imagery (p. 271-282). Springer Science + Business Media.

111. Stevenson RJ, Case TI. Olfactory dreams: phenomenology, relationship to volitional imagery and odor identification. Imagin Cogn Pers 2005; 24:69-90.

112. Weitz H, Croy I, Seo HS, Negoias S, Hummel T. Studies on Olfactory Dreaming. Chemosens Percept 2010; 3:129-134.

113. Croy I, Buschhuter D, Seo HS, Negoias S, Hummel T. Individual significance of olfaction: development of a questionnaire. Eur Arch Otorhinolaryngol. 2010; 267:67-71.

114. Seo HS, Lee S, Cho S. Relationships between personality traits and attitudes toward the sense of smell. Front Psychol 2013; 4:901.

115. Besser G, Erlacher B, Aydinkoc-Tuzcu K, et al. Body-Mass-Index Associated Differences in Ortho- and Retronasal Olfactory Function and the Individual Significance of Olfaction in Health and Disease. J Clin Med 2020; 9.

\section{Pengfei Han}

Faculty of Psychology

Southwest University

2 Tiansheng Rd.

Beibei District

400715 Chongqing

China

Tel: +862368253629

E-mail:p.han@foxmail.com 